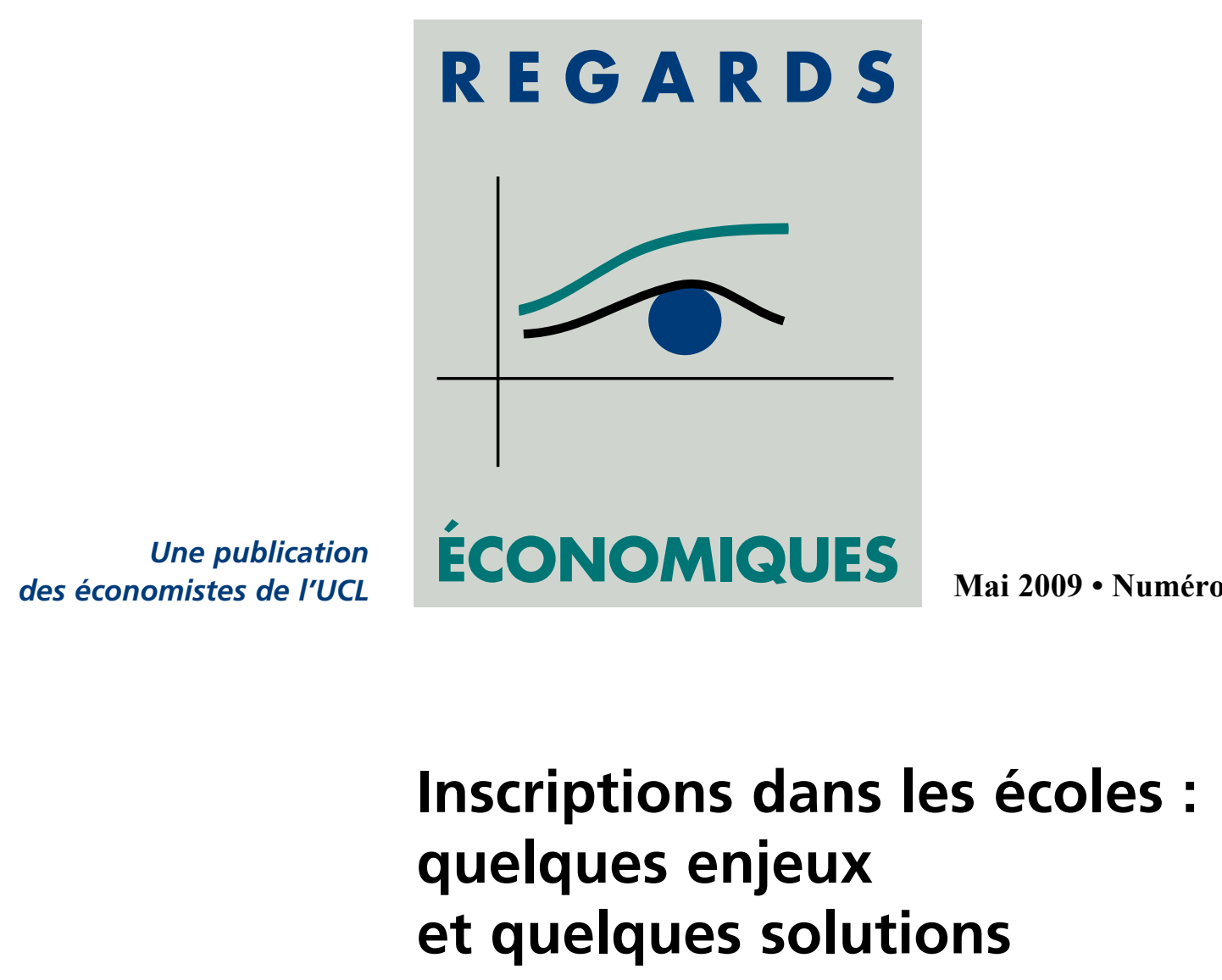

Nous présentons deux procédures d'inscription des élèves en première année de secondaire. Chaque procédure permet aux parents de révéler leurs préférences en termes d'écoles. Lorsque les préférences sont en conflit, on utilise des ordres de priorités prédéfinis. Ces ordres peuvent incarner la volonté de mixité sociale du gouvernement.

\section{François Maniquet}

Cela fait plusieurs années que l'inscription des enfants en première année de secondaire pose problème en Communauté française. L'ancien décret «inscriptions» a voulu résoudre le problème des files d'attentes devant les écoles, créé par le décret précédent, mais il a créé un autre problème aussi important : devant l'incertitude concernant la possibilité d'obtenir une inscription dans telle école de leur choix, les parents ont multiplié les demandes d'inscriptions, créant un engorgement dans un certain nombre d'écoles qui n'était pas encore résorbé au début du mois d'avril. A la mi-mars, on comptait encore à Bruxelles et en Brabant wallon quelque 2000 inscriptions multiples, alors qu'entre 1000 et 1400 enfants se trouvaient toujours sans école. La bulle se dégonfle, mais lentement, les parents ayant peur de renoncer à une inscription, craignant que cela ne les précipite, en fin de processus, vers une école qu'ils jugent moins appropriée.

Le décret voté il y a quelques jours au Parlement de la Communauté française salue l'entrée dans la législation d'une composante essentielle dans les mécanismes d'attribution des inscriptions : les préférences des parents. Même si le décret ne dit pas tout sur la manière dont ces préférences doivent être prises en compte, reconnaitre que la satisfaction des préférences des parents fait partie de l'objectif que le gouvernement doit suivre facilite la définition d'une solution juste.

La question traitée dans ce numéro de Regards économiques est celle-ci : comment, concrètement, assigner tel élève à telle école, étant donné que 1) les parents souhaitent pouvoir inscrire leurs enfants dans les écoles qui leur semblent les meilleures pour leurs enfants, 2) le pouvoir public a un objectif de mixité sociale dans les classes, et 3) les écoles ont des spécificités, des objectifs propres, qu'il convient d'essayer de respecter.

Même s'il est évident que les objectifs des parents, du pouvoir public et des écoles peuvent entrer en conflit, nous allons proposer dans cet article deux solutions concrètes au problème du choix d'école. Ces solutions concrètes, bien connues de la littérature et appliquées dans quelques pays, sont justifiées par des valeurs 
éthiques que nous définirons et discuterons. Les deux solutions sont basées sur des procédures centralisées. Chaque procédure exige que chaque famille remette un classement ordonné des différentes écoles dans lesquelles elle souhaite voir son enfant admis. La liberté de choix des parents est donc au coeur de ces solutions, de sorte que le vote du nouveau décret rend possible (et même souhaitable) l'application d'une de ces deux procédures. Chaque procédure, en outre, exige que des ordres de priorités d'élèves soient établis au niveau de chaque école. La théorie économique ne dit rien sur la manière de réaliser ces ordres de priorités. Nous expliquerons que c'est à ce niveau que doit se matérialiser le compromis entre la volonté politique de mixité sociale et les objectifs pédagogiques des écoles.

Enfin, nous décrirons comment ces deux solutions peuvent être utilisées pour améliorer la situation actuelle et augmenter la satisfaction que les parents pourront tirer de la rentrée 2009.

\section{Théorie économique de l'appariement}

Tant les files d'attentes réelles que les engorgements virtuels représentent une grande perte de bien-être. Dans un cas, il s'agit d'une perte de temps énorme, et dans l'autre il s'agit d'une incertitude parfois longue et pesante, pour, en fin de compte, ne pas être sûr d'obtenir l'accès à une école souhaitée.

Ces problèmes n'existaient pas il y a dix ans, et cela suffit à certains pour demander que l'on revienne à la situation antérieure de décentralisation extrême. Mais tout n'était pas parfait pour autant. Le problème de base venait de ce que certaines écoles, que l'on peut qualifier de populaires au sens où un grand nombre de parents souhaitaient y inscrire leurs enfants, étaient à ce point prisées que les inscriptions y étaient clôturées plus d'un an avant la rentrée scolaire. Si l'on suppose que ces écoles donnent un enseignement de bonne qualité, une telle situation signifie que cet enseignement est dispensé en priorité aux enfants dont les parents savent qu'il faut s'y prendre longtemps à l'avance, ou aux parents pouvant compter sur leurs réseaux sociaux de connaissance pour obtenir une inscription.

Bref, une telle situation rémunère une certaine information, et cette information n'est pas distribuée de façon égale dans la population. Certains diront que ce système privilégie les parents qui se démènent pour l'éducation de leurs enfants, d'autres diront qu'il privilégie les classes les mieux éduquées, qui sont aussi, évidemment, les classes sociales les plus riches. Toujours est-il qu'en fin de compte un grand nombre d'écoles rassemblent des enfants d'origine sociale homogène, avec comme conséquence une inégalité énorme en termes de performances scolaires moyennes des élèves entre écoles. Les derniers gouvernements successifs de la Communauté française, ne se satisfaisant pas de cette inégalité entre écoles, ont estimé que réaliser la mixité sociale dans les classes pouvait être un moyen de rétablir plus d'égalité entre les écoles, et, de là, entre les élèves. Si l'on accepte ce point de vue, il est impossible de se satisfaire d'une situation de laisser-faire comme celle que l'on connaissait avant les derniers décrets : le pouvoir public doit réguler les inscriptions.

Le coeur de ce texte vient de ce que le problème ainsi posé fait l'objet depuis plus de vingt ans d'une abondante littérature en science économique connue sous le nom de théorie de l'appariement ${ }^{1}$, théorie qui connaît un nombre croissant d'applications dans le domaine de la répartition des étudiants entre différents cours à option dans les universités, la répartition des étudiants en médecine entre les hôpitaux où ils doivent continuer leur formation, et, plus récemment, la réparti-

${ }^{1}$ Les bases de cette théorie ont été développées par Gale et Shapley (1962) et, surtout, Roth et Sotomayor (1990). 


\section{... Théorie économique de l'appariement}

\section{Le problème de base}

tion des élèves entre les différentes écoles d'un district scolaire (ces applications ont surtout eu lieu aux Etats-unis, en Angleterre et en Espagne).

Dans cet article, nous allons montrer comment cette littérature peut éclairer de manière décisive le problème concret et de court terme de la répartition des enfants entre les écoles, mais nous allons aussi montrer que l'application des solutions qui ont été trouvées à ce problème exige que l'on prenne des positions claires sur un certain nombre d'autres problèmes plus fondamentaux. En effet, toute solution valable au problème des inscriptions exige que l'on définisse des ordres de priorité des élèves dans les écoles. Ces ordres de priorité ne peuvent être valablement établis que si l'on s'est mis d'accord sur le type de mixité sociale que l'on veut encourager dans les écoles. En outre, il faut décider si l'on souhaite imposer la mixité aux écoles ou si, au contraire, on laisse à chaque école le choix de fixer elle-même les ordres de priorités des élèves qu'elle va accueillir.

L'objectif premier de cet article est donc de présenter les deux procédures de base qui doivent être considérées comme les meilleures solutions au problème des inscriptions. Notre position est donc qu'une solution en Communauté française doit être fondée sur une de ces deux procédures. Le second objectif est d'illustrer le fait que l'application de ces deux procédures exige qu'un certain nombre d'autres questions soient résolues. Nous ne proposerons pas de réponses à ces questions, mais nous ferons de temps en temps référence à certains enseignements de la science économique qui peuvent éclairer les décisions.

Qu'on le veuille ou non, qu'on s'en défende ou non, tout système d'inscriptions scolaires répond, de manière implicite ou explicite, à la question fondamentale suivante : lorsque deux élèves souhaitent être inscrits dans une école qui n'a qu'une place disponible, lequel des deux obtiendra l'inscription et pourquoi ??

Une solution pourrait être de mettre la place vacante aux enchères. En plus des problèmes conceptuels que cette solution soulève, elle n'est souhaitée par personne et on peut donc l'écarter. Mais toute autre solution exige que soit établi un ordre de priorité entre ces deux élèves. Par exemple, dans le système antérieur, l'ordre de priorité était déterminé sur la base du principe : premier arrivé premier inscrit. C'est le principe que les derniers gouvernements refusent, pour la raison indiquée plus haut. Notons que d'autres principes ont été appliqués par les écoles et ont été interdits par les décrets, comme par exemple donner la priorité à des élèves dont les parents avaient, en leur temps, été élèves de cette école.

La question est donc : quels principes peuvent gouverner la mise sur pied de ces listes de priorités ? Un critère clair et unanimement admis est celui de la présence de frères ou soeurs de l'élève dans l'école. C'est évidemment rationnel d'appliquer ce critère. Un second critère souvent appliqué (notamment aux Etats-Unis) est celui de la distance entre l'habitation de l'élève et l'école. Il peut être tentant de l'utiliser, par exemple si l'on pense que minimiser les trajets (en voiture) est bon pour l'environnement et donc pour la collectivité, et fait économiser du temps aux parents. Mais ce critère peut lui-même porter les germes d'une segmentation sociale bien pire que celle que l'on souhaite combattre.

En effet, il est bien établi maintenant que lorsque la localisation de l'habitation joue un rôle déterminant dans l'accès aux écoles, la régulation se fait par le choix de la localisation, de sorte que les maisons proches d'une école réputée sont visées

\footnotetext{
${ }^{2}$ Si l'on modifie la question en disant que 120 élèves souhaitent être inscrits dans une école qui n'a que 100 places disponibles, cela ne change rien au fond du problème : les nombres n'importent pas.
} 


\section{... Le problème de base}

3. Priorité à qui ? par les parents les plus soucieux d'envoyer leurs enfants dans cette école, le prix des habitations augmente, et les quartiers avoisinant l'école deviennent des quartiers homogènes de classe aisée. Ce phénomène est particulièrement remarquable dans certains districts des Etats-Unis, mais est aussi clair en France, où le critère géographique est presque le seul utilisé. La Belgique a réussi jusqu'à présent à échapper à une telle ghettoïsation. Ce serait bien dommage de l'installer, alors que l'objectif est précisément la mixité sociale. Il convient donc d'être extrêmement prudent dans l'utilisation du critère géographique.

Nous en arrivons à la question qui devrait être au coeur de la discussion : quels critères doivent jouer un rôle dans l'ordre de priorité ? Ou, pour le dire autrement : quelle mixité sociale voulons-nous ? S'agit-il d'essayer de mélanger dans les classes les élèves de groupes socio-économiques différents? S'agit-il de mélanger les élèves d'origine belge et ceux d'origine étrangère ? S'agit-il de mélanger les élèves en fonction de leur langue maternelle ? S'agit-il de mélanger les élèves en fonction de leurs performances scolaires ? Pour parler abstraitement, disons que si l'on souhaite mélanger des enfants de type A avec des enfants de type B et que les premiers représentent dix pour cent de la population, on s'assurera que les ordres de priorités de chaque école soient tels que sur les dix enfants ayant la plus grande priorité dans cette école, un soit de type A et 9 de type B, sur les dix enfants ayant les ordres de priorités de 11 à 20, on trouve aussi un enfant de type A et 9 de type B, etc. Notons d'emblée que cela ne signifie pas qu'en fin de compte chaque école soit peuplée de 10 pour cent d'enfants de type A et de 90 pour cent d'enfants de type B, comme nous le verrons plus bas (c'est une conséquence du fait que l'on tient compte des préférences des parents).

Il est urgent que l'on sache quel est l'objectif de mixité sociale à poursuivre en Communauté française. En outre, il serait bien difficile de mettre en oeuvre une politique qui n'aurait pas un minimum de consensus parmi tous les acteurs, en particulier parmi les écoles, nous y reviendrons.

Avant de décrire comment un objectif de mixité sociale, couplé au respect des objectifs des écoles, et tenant compte des préférences des parents peut donner lieu à une procédure claire d'inscriptions, il nous paraît utile de soulever la question suivante : est-ce que la mixité dans les classes est un bon moyen d'améliorer la qualité de notre enseignement, en particulier de l'enseignement des enfants défavorisés, quelle que soit notre vision de qui est favorisé et qui ne l'est pas ? Nous voudrions passer en revue quelques arguments du débat, dans le seul objectif de voir si la littérature économique peut les éclairer.

Un premier argument en faveur de la mixité sociale dans les classes pourrait être fondé sur le «peer effect» : on dit qu'il y a un «peer effect» lorsque les performances scolaires d'un enfant sont affectées par la composition de sa classe (en terme de niveau scolaire, origine socio-économique, origine ethnique, etc. des autres élèves). On peut imaginer plusieurs types de «peer effects» : quelques élèves brillants peuvent tirer les autres élèves de la classe vers le haut, ou, au contraire, en leur faisant perdre confiance en eux, faire baisser leurs performances scolaires; des élèves turbulents, ou des élèves dont les performances scolaires sont moindres, peuvent distraire les autres élèves de la classe et faire baisser leurs résultats scolaires. Mélanger les types d'élèves au sein de la classe peut mener tout le monde vers le haut grâce au partage de leurs connaissances propres, ou au contraire, mener tout le monde vers le bas vu la difficulté de trouver un langage commun. On le voit, l'existence et la nature d'un «peer effect» n'est pas une question théorique (on pourrait argumenter dans tous les sens) mais une question empirique. 


\section{... Priorité à qui ?}

La littérature sur cette question est abondante. L'objectif principal de cette littérature est d'estimer le rôle joué par cet effet dans les performances scolaires des élèves, parmi les autres variables susceptibles d'influencer les résultats (capacités intrinsèques des élèves, qualité des professeurs, qualité de l'école, aide des parents, etc.). Après des dizaines d'années de recherche dans ce domaine, il est juste de dire qu'aucune conclusion définitive n'est tirée. En particulier, si l'on s'intéresse à l'effet que peut avoir l'hétérogénéité de la composition de la classe sur les performances d'un élève, en fonction des données disponibles, en fonction des méthodes statistiques utilisées, on observe tantôt un «peer effect» positif, tantôt négatif, tantôt nul. ${ }^{3}$ Justifier la mixité sociale dans la classe sur cette base semble donc bien risqué.

Un deuxième argument consiste à dire qu'indépendamment de leur population scolaire les écoles ne se valent pas, certaines étant plus efficaces que d'autres dans leur mission d'éducation. De ce point de vue, l'accès à une «bonne» école est un bien rare, et l'objectif de la mixité sociale est de donner à chaque enfant la même chance d'avoir accès à ce bien rare. Il y a également une littérature sur la qualité des écoles, au sens de l'effet plus ou moins grand qu'elles peuvent avoir sur les résultats des élèves. A nouveau, mesurer cet effet est extrêmement compliqué, dans la mesure où, comme pour l'évaluation du «peer effect», il convient de différencier avec précision cet effet d'autres effets (au nombre desquels, à nouveau, les capacités intrinsèques des élèves, l'aide des parents, etc.). Ici, les résultats les plus fréquents indiquent un effet positif de la qualité de l'école sur les performances des élèves (la qualité est souvent mesurée en terme d'autonomie de gestion des écoles, d'infrastructure scolaire, de la qualité des enseignants, etc.). ${ }^{4}$ Cet argument peut donc justifier que l'on mélange les publics scolaires, mais seulement si le «peer effect» lié à la mixité dans la classe n'est pas négatif.

Un troisième argument consiste à dire que les élèves sont plus ou moins difficiles à scolariser, et que l'objectif de la répartition des élèves entre écoles serait de répartir plus également le poids des élèves difficiles entre les enseignants. Cet objectif ne peut justifier la mixité socio-économique (ou ethnique, etc.) que s'il y a une corrélation entre être difficile à scolariser et appartenir à telle ou telle classe socio-économique (ou telle ou telle ethnie, etc.). Par ailleurs, affirmer que certains enfants sont plus difficiles à scolariser n'a, certes, pas besoin de longues preuves, mais on peut pourtant rétorquer qu'enseigner à un public difficile requiert des aptitudes particulières que seuls quelques enseignants ont et il est plus efficace de concentrer le public difficile dans les classes de ces enseignants (tout en donnant à ces enseignants les moyens de mettre en oeuvre les méthodes scolaires adéquates).

Certains arguments du débat s'opposent directement à la mixité. Un quatrième argument consiste à dire que la mixité sociale n'est pas la meilleure manière d'augmenter les chances de bonne scolarisation des enfants des classes précaires. Selon cet argument, des écoles socialement homogènes permettent aux gouvernements de financer de manière différenciée ces écoles, en donnant des moyens supplémentaires à celles qui accueillent des élèves moins favorisés. Cet argument semble avoir trouvé écho chez nous puisque le financement différencié existe depuis plusieurs années et vient d'être renforcé par un décret récent.

On le voit, il n'est pas évident qu'instaurer la mixité dans les classes est la meilleure manière d'augmenter les chances de bonnes performances scolaires des élèves les moins favorisés, que ceux-ci soient définis en termes de capacité

\footnotetext{
${ }^{3}$ Voir Hoxby et Weingarth (2005) pour une présentation générale de la littérature récente. Voir aussi Hanushek (1972, 1979), Zimmer et Toma (2000) et Hanushek et al. (2003) pour des exemples d'études aux conclusions différentes.

${ }^{4}$ Voir, par exemple, Hanushek et al. (2005).
} 


\section{... Priorité à qui ?}

scolaire intrinsèque, de statut socio-économique, d'origine ethnique, etc. Il y a bien d'autres arguments à ce débat, mais ceux-ci suffisent pour montrer qu'une justification rigoureuse est nécessaire si l'on veut la mixité sociale, qu'il faut être clair sur les raisons de ce choix et donc ses objectifs, sans quoi il est impossible de fixer des priorités cohérentes dans le problème de la répartition des élèves entre les écoles.

Une remarque s'impose. Comme nous allons le voir dans les sections suivantes, la manière de comprendre la mixité sociale, et la manière d'en déduire des priorités ne va jamais à l'encontre de la volonté des parents, dans la mesure où ces priorités ne jouent qu'un rôle secondaire par rapport aux préférences des parents. Autrement dit, s'il n'y a pas de conflit entre les volontés des parents, c'est-à-dire s'il est possible de donner à chaque parent accès à l'école de son premier choix, les priorités reflétant la politique gouvernementale n'influencent en rien l'appariement final. L'objectif de mélanger les enfants d'origines socio-économiques différentes dans la même classe, par exemple, n'aura d'effet que si les parents d'origines socio-économiques différentes souhaitent envoyer leurs enfants dans les mêmes écoles et si ces écoles s'en trouvent congestionnées.

Par contre, la mixité sociale peut entrer en conflit avec les objectifs propres des différentes écoles. Cela signifie que si deux familles souhaitent inscrire leurs enfants dans la même école, alors qu'une seule place est laissée vacante, il se peut que la priorité conforme à l'objectif de mixité sociale soit différente de celle que l'école aimerait proposer. Cela vient de ce que les écoles, pour toutes une série de raisons, peuvent développer des préférences pour tel ou tel type d'élèves. Même s'il est clair que toutes ces préférences ne sont pas légitimes d'un point de vue social (comment justifier, par exemple, de donner la priorité aux enfants dont les parents furent en leur temps élèves de l'établissement), il est tout aussi clair que certaines d'entre elles peuvent parfaitement l'être d'un point de vue pédagogique. Pensons par exemple aux écoles secondaires engagées dans des projets communs avec une école primaire proche, dont l'objectif est de favoriser une transition harmonieuse d'un niveau vers l'autre. De manière plus générale, il peut être utile ici de rappeler que l'autonomie dont dispose les chefs d'établissement a été souvent épinglée comme une variable cruciale de la qualité des écoles, variable qui ellemême a un effet positif sur les performances scolaires des élèves. ${ }^{5}$

Nous ne pouvons ici que plaider pour une concertation entre les décideurs politiques à qui il revient d'énoncer clairement les objectifs de mixité sociale qu'ils souhaitent poursuivre et les établissements scolaires qui sont les mieux placés pour que la mise en oeuvre des objectifs de mixité sociale ne mette pas en péril la réalisation de leurs objectifs pédagogiques. Il devrait découler de cette concertation une manière de réaliser les ordres de priorités des enfants dans les écoles.

Supposons que des ordres de priorité ont été définis pour chaque enfant dans chaque école. Comment les utilise-t-on pour répartir les élèves, c'est la question à laquelle répond la théorie de l'appariement.

Illustrons cette théorie à travers un exemple simple. Imaginons que la population scolaire se compose de trois élèves et de trois écoles, et que chaque école dispose d'une seule place. L'ingrédient le plus important est bien sûr la volonté des parents d'envoyer leur enfant dans telle ou telle école, c'est ce que nous appellerons leurs préférences. Dans le tableau ci-dessous, les préférences de la famille 1 sont décri-

\section{Trois principes de justice}


tes dans la première colonne, et signifient que l'école qui leur semble la meilleure est l'école $A$ suivie de l'école $B$ et enfin $C$. La famille 2 estime aussi que l'école $A$ est la plus adaptée, mais $C$ est considérée meilleure que $B$ et la famille 3 a des préférences différentes, indiquées dans le tableau 1.

Tableau 1 : Préférences des familles et priorités des écoles

\begin{tabular}{lcccccc} 
& \multicolumn{3}{c}{ Elèves } & \multicolumn{3}{c}{ Écoles } \\
\hline & I & 2 & 3 & A & B & C \\
\hline $1^{\text {er choix }}$ & A & A & C & 3 & 2 & 1 ou 2 \\
$2^{\text {e choix }}$ & B & C & A & 1 & 1 ou 3 & 3 \\
$3^{\text {ee choix }}$ & C & B & B & 2 & &
\end{tabular}

D'un autre côté, les écoles ont leur liste de priorités : ainsi, l'école $A$ donne la priorité à l'élève 3 , suivi par 1 et enfin, 2 , alors que l'école $B$ donne la priorité à 2 mais ne fait pas de distinction entre 1 et 3 . Le fait qu'un ordre de priorités ne donne pas un rangement strict de tous les élèves mais laisse des classes d'équivalence est un fait essentiel sur lequel nous reviendrons plus loin.

Cet exemple nous permettra d'illustrer les difficultés qui apparaissent lorsque l'on vise à établir une procédure d'appariement des élèves aux écoles, et les solutions principales qui ont été proposées. On peut voir que le point de départ du problème ainsi illustré est lié au fait que les familles 1 et 2 aimeraient toutes les deux avoir accès à l'école $A$. Pourtant, une seule des deux, au plus, pourra y inscrire son enfant. Laquelle ? Où ira l'enfant qui se sera vu refuser l'accès à son école préférée?

Un petit calcul indique qu'il y a 6 appariements possibles. Si l'on multiplie les élèves et les écoles, on arrive rapidement à des nombres très élevés. Comment répartir les élèves entre les écoles, ou, plus exactement, comment définir une bonne procédure d'appariement?

La littérature a retenu trois grands principes de justice à l'aide desquels on doit pouvoir définir une bonne procédure :

- le respect des préférences des familles : on ne peut recommander un certain appariement que s'il est impossible de faire mieux pour toutes les familles;

- le respect des priorités : la seule raison pour laquelle une famille peut se voir refuser une place dans une école, c'est que toutes les places ont été allouées à des familles ayant une plus grande priorité dans cette école;

- le respect de la vérité : chaque famille doit avoir intérêt à révéler correctement ses préférences en matière d'écoles; autrement dit, aucune famille ne peut gagner, au sens d'obtenir une inscription dans une école qu'elle estime meilleure, en annonçant des préférences qui ne correspondraient pas à ses véritables choix.

Passons en revue ces trois principes, en les illustrant à partir de l'exemple ci-dessus et en présentant leurs principales justifications.

Dans l'exemple, l'appariement $B C A$, qui se lit «l'élève 1 est admis dans l'école $B$, 2 dans $C$ et 3 dans $A »$, ne respecte pas les préférences des familles. En effet, il est possible de faire mieux, au sens où il existe un autre appariement tel que personne n'y perd, alors que certains sont plus satisfaits : il s'agit de l'appariement $B A C$. L'élève 1 n'y perd pas, alors que 2 et 3 sont maintenant admis dans des écoles qu'ils estiment meilleures pour eux. Quelques remarques peuvent être faites :

- l'amélioration qui s'est produite lorsque l'on est passé de l'appariement $B C A$ à 


\section{... Trois principes de justice}

\section{Un dilemme, deux solutions}

L'enseignement de base que l'on doit à la théorie de l'appariement est la preuve qu'il n'existe aucune procédure de répartition des élèves dans les écoles qui satisfasse ces trois principes. Cela signifie qu'un choix politique important doit être opéré : les décideurs doivent renoncer à un principe (au moins). Par ailleurs, on peut également démontrer que si l'on perd le principe de respect de la vérité, alors les comportements des familles deviennent difficilement prévisibles et il est vraisemblable que l'on perde également l'un ou l'autre des deux autres principes sans le savoir.

Le choix est donc entre d'une part s'attacher au respect des préférences des familles, et d'autre part s'attacher au respect des priorités. Dans chacun des cas, la théorie a mis en évidence une bonne procédure, que nous expliquons ci-dessous.

Si l'on est intéressé par le respect des préférences des familles et le respect de la vérité, il faut utiliser la procédure des cycles d'échange des priorités. Illustrons cette procédure dans le cas de notre exemple. La première chose à faire est d'af- 
finer les ordres de priorité des écoles pour obtenir un classement strict de tous les élèves. Cela peut se faire par n'importe quel système de tirage au sort. Supposons que cela donne la situation décrite dans le tableau 2.

Tableau 2 : Préférences des familles et priorités affinées des écoles

\begin{tabular}{lcccccc} 
& \multicolumn{3}{c}{ Elèves } & \multicolumn{3}{c}{ Écoles } \\
\hline & I & 2 & 3 & A & B & C \\
\hline $1^{\text {er choix }}$ & A & A & C & 3 & 2 & 1 \\
$2^{\text {e choix }}$ & B & C & A & 1 & 3 & 2 \\
$3^{\text {e c choix }}$ & C & B & B & 2 & 1 & 3
\end{tabular}

A partir des préférences que les familles ont révélées, on regarde l'école que chaque famille préfère, et l'élève à qui chaque école a donné la priorité. Nous obtenons donc que les élèves 1 et 3 préfèrent les écoles $A$ et $C$ ( 1 préfère $A$ et 3 préfère $C$ et, en même temps, sont prioritaires dans ces écoles : 1 est prioritaire chez $C$ et $3 \mathrm{chez} A$ ). On inscrit alors 1 à l'école $A$ et 3 à l'école $C$, ce qui revient à dire que 1 et 3 ont échangé leurs priorités. Enfin, 2 est admis à l'école $B$.

Si l'on est intéressé par le respect des priorités et de la vérité, il faut utiliser la procédure de l'acceptation différée. Illustrons à nouveau cette procédure dans le cas de notre exemple. A partir des préférences que les familles ont révélées, on regarde l'école que chaque famille préfère, en considérant que chaque famille demande une inscription dans cette école : 1 demande une inscription dans l'école $A, 2$ aussi, et 3 demande une inscription en $C$. Ensuite, chaque école ayant reçu plus de demandes que le nombre de places disponibles sélectionne le nombre correct d'élèves en fonction de ses priorités. Dans l'exemple, $A$ qui a reçu deux demandes pour une place, sélectionne 1 , qui a priorité sur 2 . Nous disons alors que 1 est provisoirement inscrit dans $A$, et 3 dans $C$. Ensuite, tous ceux qui ne sont pas encore inscrits provisoirement, dans notre cas, l'élève 2, demande une inscription dans la meilleure école pour lui, parmi celles qui ne l'ont pas encore refusé. Ici, il demande une inscription en $C$, qui se retrouve avec deux demandes, et choisit 2. Nous voyons donc que 3, qui était inscrit provisoirement en $C$, est maintenant écarté de cette école au profit de 2 , puisque celui-ci y détient une priorité supérieure. C'est au tour de 3 de faire une nouvelle demande, qu'il adresse à $A$, et ainsi de suite. La théorie montre que cette procédure mène toujours à un résultat, et un résultat unique. Dans notre exemple, il s'agit de l'appariement $B C A$. Observons que cet appariement ne respecte pas les préférences des familles, comme nous l'avons annoncé plus haut : l'appariement $B A C$ est meilleur pour 2 et 3 , qui accepteraient donc volontiers d'échanger leurs places.

Plusieurs remarques importantes doivent être faites.

- Tout d'abord, ces deux procédures sont des procédures centralisées : il faut que les familles s'adressent à une autorité centrale, et non directement aux écoles. Concrètement, dans les pays où ces procédures sont appliquées, les familles remplissent un formulaire dans lequel elles donnent leurs ordres de préférence sur un nombre déterminé d'écoles. Ce nombre doit être calculé de telle sorte que l'information ainsi transmise soit suffisante pour répartir les élèves (s'il est connu, par exemple, que 6 écoles sont systématiquement engorgées, il est indispensable de demander aux parents de ranger plus de 6 écoles). L'utilisation d'un mécanisme centralisé doit être vu comme un moindre mal pour résoudre le problème de l'inscription des élèves lorsque les contraintes matérielles ne permettent pas que chaque famille ait accès à l'école qu'elle préfère. D'un côté les parents préfé- 
... Un dilemme, deux solutions reraient sans doute que l'inscription suive un contact personnel avec le directeur de l'école ou un de ses collaborateurs. D'un autre côté les directeurs d'école peuvent avoir l'impression de perdre la maîtrise d'un moment essentiel de la vie d'une école. Rappelons d'une part que l'objectif poursuivi est bien un objectif de justice sociale, qui exige de traiter de manière égale, et donc anonyme, toutes les familles. D'autre part, si les ordres de priorités sont effectivement réalisés dans un esprit de coopération entre les décideurs politiques et les directeurs d'école, ceuxci peuvent garder une influence importante sur le résultat de la procédure.

- Ensuite, ces procédures nécessitent que chaque école ait un ordre strict de priorité sur l'ensemble des élèves. Cela exige soit que les critères utilisés pour constituer cet ordre, qui, rappelons-le, incluent les critères par lesquels le gouvernement souhaite mettre en oeuvre la mixité sociale, débouchent sur un ordre strict, ce qui est extrêmement peu vraisemblable, soit que l'on ait recours à un système de loterie. Le slogan selon lequel «l'avenir de nos enfants ne peut se jouer sur une loterie» signifie sans doute que les critères appliqués au moment de la définition des priorités devraient permettre de départager n'importe quelle paire d'élèves. C'est malheureusement extrêmement difficile à concevoir. Comment, en effet, départager deux enfants du même quartier, de même statut socio-économique, de même ethnie, etc. et dont les parents ont les mêmes préférences en termes d'écoles ? Il faut se rendre à l'évidence : le tirage au sort est une solution acceptable pour départager les ex-aequo de manière juste.

- Enfin, que l'on utilise l'une ou l'autre des solutions proposées, la mixité sociale ne sera le résultat du processus que si les gens la veulent eux-mêmes. En effet, quels que soient les critères de priorité utilisés, ils ne jouent de rôle que lorsque les préférences des familles sont en conflit. Par conséquent, le grand avantage de ces procédures est de respecter de manière absolue la liberté de choix des familles, et de n'intervenir qu'en cas d'engorgement dans les écoles : ces procédures refusent de faire soi-disant le bonheur des gens contre leur volonté. Il faut donc bien comprendre que l'usage des loteries ne signifie pas que le mécanisme centralisé se permet d'envoyer n'importe quel enfant dans n'importe quelle école au hasard. Au contraire, la loterie n'intervient que lorsque ni les préférences des parents ni les ordres de priorités des écoles ne permettent de déterminer à quelle école chaque enfant aura accès.

Nous pouvons tirer quelques conclusions sur les décisions à prendre à court terme et à moyen terme. A court terme, c'est-à-dire pour la rentrée prochaine, deux problèmes se posent. D'abord, certains parents ne savent toujours pas où iront leurs enfants, vu qu'ils les ont provisoirement inscrits dans plusieurs écoles, que ces écoles ont des listes d'attente et que les engorgements n'ont pas été réglés. C'est particulièrement le cas à Bruxelles.

Des procédures se mettent en place actuellement pour régler ce problème d'engorgement, en essayant de satisfaire au mieux les parents, mais elles ont malheureusement toutes le problème de l'absence de respect de la vérité, au sens où nous l'avons défini ici. En effet, on ne peut attendre des familles qu'elles révèlent correctement leurs préférences si elles ne savent pas avec précision comment l'information qu'elles révèlent sera traduite en un choix d'école.

Ensuite, de nombreux parents ont une inscription ferme dans une école, mais cette école n'est pas leur premier choix, et ils seraient prêts à échanger leur inscription avec celle d'un autre élève.

La solution des cycles d'échange des priorités définies plus haut peut apporter une solution à ces deux problèmes. Il s'agit d'une part de rompre les inscriptions multiples en utilisant des ordres de priorité, éventuellement déterminés par loterie. 


\section{... Quelques recommandations}

Une fois cette étape terminée, c'est-à-dire une fois que chaque élève aura reçu son affectation, il conviendra de laisser les élèves échanger leurs inscriptions selon une procédure similaire à celle des cycles d'échange des priorités.

Bien sûr, ce processus d'échange ne se fait pas concrètement entre les parents : chaque famille annonce l'ordre des écoles qu'elle préfère à l'école dans laquelle elle a obtenu une inscription, et la procédure centralisée applique une variante de la procédure décrite ci-dessus (dont les détails doivent encore être déterminés). Une telle procédure respecte la vérité au sens défini plus haut, elle respecte les préférences des familles, et elle garantit à chaque famille d'obtenir une inscription dans une école qu'elle estime au moins aussi bonne que celle dans laquelle elle a actuellement une inscription.

A moyen terme, un choix doit être fait entre les deux procédures décrites ci-dessus, et la procédure de base doit être adaptée à la réalité de notre Communauté (il faut par exemple, surtout à Bruxelles, tenir compte de la possibilité pour certains de préférer une école de la Communauté flamande à certaines écoles de la Communauté française).

Comme nous l'avons expliqué, cela exige de mettre au point une manière de construire les ordres de priorités. Si ceux-ci ne visent pas la mixité sociale, alors les priorités classiques liées notamment à la présence de frères ou soeurs dans l'école doivent être complétées par un système de loterie.

$\mathrm{Si}$, au contraire, ceux-ci visent une certaine mixité sociale dans les classes, alors il convient d'énoncer des principes clairs, et de les faire connaître. Il faut se rendre compte qu'en fonction des préférences des familles, de tels ordres de priorités destinés à bouleverser l'équilibre actuel des populations scolaires peuvent avoir des conséquences considérables sur ces populations, et donc sur l'effort pédagogique des enseignants, qui devront s'adapter à un public nouveau. Cela justifie d'une part que les écoles soient associées à l'élaboration de ces ordres de priorités, et d'autre part que le changement se fasse lentement et dans la douceur. Trop de réformes dans notre système scolaire se sont heurtées à des difficultés liées d'une façon ou d'une autre au manque de concertation du corps enseignant et des directeurs d'école. ${ }^{6}$ Pourquoi même ne pas penser à une période d'expérimentation, où l'une ou l'autre sous-région servirait de région test, ce qui permettrait d'évaluer les réformes avant de, peut-être, les généraliser à l'ensemble de la Communauté ? 


\section{Références}

Directeur de la publication :

Vincent Bodart

Rédactrice en chef :

Muriel Dejemeppe

Comité de rédaction : Paul Belleflamme,

Vincent Bodart, Thierry Bréchet,

Muriel Dejemeppe, Frédéric Docquier,

Jean Hindriks, François Maniquet,

Marthe Nyssens

Secrétariat \& logistique : Anne Davister

Graphiste : Dominos
Hugues Draelants, 2009. Réforme pédagogique et légitimation. Le cas d'une politique de lutte contre le redoublement. Bruxelles : de Boeck Université.

David Gale and Lloyd S. Shapley, 1962. College admissions and the stability of marriage. American Mathematical Monthly 69, pp. 9-15.

Eric A. Hanushek, 1972. Education and race : an analysis of the educational production process. Health-Lexington : Cambridge, MA.

Eric A. Hanushek, 1979. Conceptual and empirical issues in the estimation of educational production functions. Journal of Human Resources, 14, pp. 351-388.

Eric A. Hanushek, John F. Kain, Jacob M. Markman and Steven G. Rivkin, 2003. Does peer ability affect student achievement? Journal of Applied Econometrics, 18, pp. 527-544.

Eric A. Hanushek, Steven G. Rivkin and John F. Kain, 2005. Teachers, schools, and academic achievement. Econometrica 73(2), pp. 417-458.

Caroline M. Hoxby and Gretchen Weingarth, 2005, Taking race out of the equation : School reassignment and the structure of peer effects, unpublished manuscript.

Alvin E. Roth and Marilda A. Oliveira Sotomayor, 1990. Two-sided matching : A study in game-gheoretic modeling and analysis. Econometric Society Monograph. Cambridged University Press : Cambridge.

Marijn Verschelde, Jean Hindriks, Glenn Rayp, Koen Schoors, 2009. Explaining regional disparity in education in Begium - a R.E.S.T. approach : first robust results. Unpublished manuscript.

Ron W. Zimmer and Eugenia F. Toma, 2000. Peer effects in private and public schools across countries. Journal of Policy Analysis and Management 19, pp. 75-92.
Regards Économiques a le soutien financier du Fonds de la Recherche Scientifique - FNRS.

IRES-UCL

Place Montesquieu, 3

B1348 Louvain-la-Neuve

http://www.uclouvain.be/regardseconomiques

regard-ires@uclouvain.be.

tél. 010/47 3426 